\title{
Transcatheter mitral valve replacement for degenerated mitral valve bioprostheses, failure of mitral valvuloplasty and native valve with severe mitral annulus calcification: a systematic review and meta-analysis
}

Tao You ${ }^{1,2^{*}+}$, Wei Wang ${ }^{2,3+}$, Kang Yi ${ }^{1,2}$, Jie Gao ${ }^{2,3}$, Xin Zhang ${ }^{2,4}$, Shao-E. He ${ }^{2,5}$, Xiao-Min Xu ${ }^{2,3}$, Yu-Hu Ma ${ }^{2,3}$ and Xin-Yao Li ${ }^{2,3}$

\begin{abstract}
Background: Although transcatheter technology has achieved some success in the field of mitral valves, the feasibility of applying it to patients with degenerated mitral valve bioprostheses (valve-in-valve, ViV), failure of mitral valvuloplasty (valve-in-ring, ViR) and serious mitral annulus calcification (vale-in-MAC, ViMAC) has not been effectively evaluated.

Methods: By searching published literature before December 5, 2020 in four databases, we found all the literature related to the evaluation of feasibility assessment of TMViV, TMViR and TMViMAC. Outcomes focused on all-cause mortality within 30 days, bleeding and LVOT obstruction.

Results: A total of six studies were included, and all of them were followed up for at least 30 days. After analysis of the ViV-ViR group, we obtained the following results: the all-cause mortality within 30 days of the ViV group was lower than that of the ViR group. Life-threatening or fatal bleeding was more likely to occur in the ViR group after surgery. At the same time, the ViR group was more prone to left ventricular outflow tract obstruction. However, in the ViMAC-ViR group, only the all-cause mortality within 30 days and stroke were statistically significant. In the indirect comparison, we found that TMViV had the best applicability, followed by TMViR. There were few TMViMAC available for analysis, and it requires further studies to improve the accuracy of the results.
\end{abstract}

Conclusion: TMViV and TMViR had good applicability and could benefit patients who underwent repeat valve surgery. The feasibility of TMViMAC needs to be further explored and improved.

Keywords: Mitral valve, TMVR, Valve-in-valve, Valve-in-ring, Valve-in-MAC, Systematic review, Meta-analysis

*Correspondence: youtao2016@126.com

${ }^{\dagger}$ Tao You and Wei Wang contributed equally to this work

${ }^{1}$ Department of Cardiovascular Surgery, Gansu Provincial Hospital, No. 204, Donggang West Road, Lanzhou City 730000, Gansu Province, China

Full list of author information is available at the end of the article

\section{Introduction}

Mitral valve disease is an abnormal valve structure or function caused by mucoid degeneration, congenital disease, degenerative disease and inflammation. From the latest American Heart Association (AHA) statistics, the incidence and mortality of mitral valve disease are increasing annually [1]. Patients suffering from severe original author(s) and the source, provide a link to the Creative Commons licence, and indicate if changes were made. The images or other third party material in this article are included in the article's Creative Commons licence, unless indicated otherwise in a credit line to the material. If material is not included in the article's Creative Commons licence and your intended use is not permitted by statutory regulation or exceeds the permitted use, you will need to obtain permission directly from the copyright holder. To view a copy of this licence, visit http://creativecommons.org/licenses/by/4.0/. The Creative Commons Public Domain Dedication waiver (http://creativeco mmons.org/publicdomain/zero/1.0/) applies to the data made available in this article, unless otherwise stated in a credit line to the data. 
mitral valve disease (insufficiency, regurgitation) were increasingly treated with annuloplasty rings or prosthetic biological valves. By analysing the data of heart valve replacement patients in California, USA, from 1996 to 2013, it was found that during this period, the utilization rate of bioprostheses during mitral valve replacement increased from 16.8 to $53.7 \%$ [2]. Due to tissue degeneration and disease progression, bioprosthetic tissue valves and natural valves that have undergone surgical repair are prone to degenerate and form lesions over time, and the vast majority of patients will require another operation [3-6]. From the current perspective, the number of repeated mitral valve operations in various heart centres around the world is increasing, and with the addition of experience, various postoperative curative effects are constantly improving. However, the risk of repeated mitral valve surgery remains higher than that of the first mitral valve surgery. Several reports have shown that the risk of repeated mitral valve surgery is very high. The 30-day mortality rate for elective mitral valve surgery was between 6.3 and $15 \%$, and the mortality rate for emergency surgery was $17.8 \%$ [7-10]. When the third or fourth operation was required, the 30-day mortality rates for elective operations were $17.3 \%$ and $40 \%$, respectively, while emergency operations were $40 \%$ and $44 \%$ [11]. In recent years, transcatheter mitral valve replacement (TMVR) has become an alternative to traditional cardiac surgery, and it is often used in patients with severe mitral valve disease, such as severe mitral valve bioprosthesis degradation, failure of valvuloplasty surgery, or severe mitral valve natural annulus calcification [12,13]. Recent studies have shown that TMVR is the first choice of treatment for patients with repeated mitral valve surgery and high-risk mitral valve disease who are not suitable for traditional surgery [14].

The degenerative changes of the mitral valve bioprosthesis (valve-in-valve, $\mathrm{ViV}$ ) and the failure of surgical rings (valve-in-ring, $\mathrm{ViR}$ ) were largely due to the rise in life expectancy of the elderly and the short-term durability of bioprostheses compared to the mechanical mitral valve $[15,16]$. After Cheung first reported transcatheter mitral valve-in-valve (TMViV) implantation in 2009 [17] and De Weger performed transcatheter mitral valve-inring (TMViR) replacement for the first time in 2011 [18], an increasing number of patients received these two types of surgery and benefited from them. Compared with traditional surgery, patients have achieved some efficacy after using TMVR. Nevertheless, the ultimate results remain unsatisfactory due to the patients' relatively poor baseline characteristics and various comorbidities, especially patients who received transcatheter mitral valve-in-mitral annulus calcification (TMViMAC) [15]. At one time, some clinicians doubted the feasibility of TMVR. The earliest experience of TMVR with severe mitral annulus calcification (MAC) was collected in the TMVR of the MAC Global Registry, reporting a mortality rate of $25 \%$ at 30 days [19]. A follow-up study from the multicentre TMVR registry reported a 30-day mortality rate of $34.5 \%$ [20]. In the existing reports, we found that the relatively high mortality rate was due to severe comorbidities and technical challenges related to calcium load [20,21]. Although the use of transcatheter mitral valve replacement for patients with severe mitral valve ring calcification still had a high mortality rate, it must be admitted that compared with traditional mitral valve surgery, TMVR has become an urgent and preferred treatment for high-risk severe mitral valve disease.

With the development of the catheter era, it is necessary to evaluate the feasibility, pros and cons of TMVR for degenerated mitral valve bioprostheses, mitral valvuloplasty failure and serious mitral annulus calcification. From the first report to the present [22], many studies have been published, and there are also some authoritative statistical results from multiple centres. However, to the best of our knowledge, there have been no systematic reviews assessing the early postoperative mortality and complications of TMVR for degenerated mitral bioprostheses (ViVs), failed surgical rings (ViRs), and native valves with severe mitral annular calcification (ViMAC). In this article, we performed a meta-analysis and systematic review on the results of ViV, ViR and ViMAC to provide a reference for the selection of operation methods for patients with indications.

\section{Material and methods}

\section{Protocol and registration}

This systematic review and meta-analysis was performed according to the preferred reporting items for systematic reviews and meta-analyses (PRISMA) statement. The protocol was registered on INPLASY (202130113) and is available in full on inplasy.com (https://inplasy.com/inpla sy-2021-3-0113).

\section{Publication selection}

The search terms were determined through the "PICO" principle, systematic electronic searches were conducted in PubMed, Embase, Web of Science and the Cochrane Library, and the references of the included documents were manually searched to identify other publications. The time was from the establishment of the database to December 5, 2020. The purpose was to find all relevant documents on transcatheter mitral ViV, ViR and ViMAC.

Search terms were Valve-in-Ring, Valve in Ring, ViR, Valve-in-Valve, Valve in Valve, ViV, Valve-in-Mitral Annular Calcification, Valve in Mitral Annular Calcification, ViMAC. 
The retrieval strategy is shown below using the Web of Science as an example:

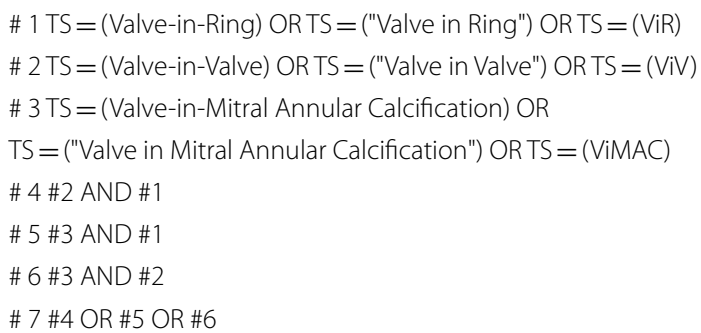

\section{Inclusion and exclusion criteria} Inclusion criteria

1. Articles written in English. 2. Minimum of 30 days follow up post-procedure. 3 . The subject of the study was the outcomes of TMVR for patients with degenerated bioprostheses [valve-in-valve (ViV)], failed annuloplasty rings [valve-in-ring (ViR)], and severe mitral annular calcification [valve-in-mitral annular calcification (ViMAC)]. 4. The research included $\geq 10$ patients undergoing either ViV-ViR, ViR-ViMAC or ViV-ViR-ViMAC.

\section{Exclusion criteria}

1. Meeting abstracts, comments, case reports, letters and expert opinions. 2. Duplicate publication of data or unable to extract data. 3. Except for mitral annular calcification, TMVR represents the native mitral valve. 4 . The study lacks main details about postprocedure results. 5 . Animal-based studies.

\section{Data extraction and quality assessment}

When we determined the final inclusion of the literature, we carefully read the full text and extracted the following data: general information of the literature (first author, publication time and country); baseline characteristics such as age, female sex, diabetes mellitus, hypertension, atrial fibrillation and 14 other pieces of information; allcause mortality, bleeding and the other nine major recent outcome indicators (within 30 days).

All the included literature was evaluated from three aspects through the Newcastle-Ottawa Scale (NOS) scoring standard: population selection, comparability and outcome. There were nine questions in total, and the highest score was 9 points. It was generally believed that when the score was $\geq 7$, the study was considered high quality [23]. Among the scoring items, except for the fifth scoring standard, which could be up to two points, the other items were all one point [24].

This part was independently conducted and crosschecked by two researchers and discussed and resolved in case of differences.

\section{Statistical methods and data processing}

All analyses were conducted using RevMan 5.4 (http:// ims.cochrane.org/revman) [Computer program]. We chose unadjusted raw data because various studies have not adjusted for the same set of confounding factors. Categorical variables are expressed as the number of occurrences, and the effect measure was the odds ratio (OR). Continuous variables are expressed as the mean \pm SD. When the unit of measurement was consistent, the mean difference (MD) was used; otherwise, the mean difference (SMD) was used. A standard confidence interval of $95 \%$ (95\% CI) was applied in all analyses. The $\mathrm{Q}$ and $I^{2}$ tests were used for statistical heterogeneity analysis. When $I^{2}>50 \%$ or $P<0.1$, the random effects model was adopted; if not, the fixed effects model was adopted. The test level $\alpha=0.05$, which means that when the $P$ value is $<0.05$, it is considered statistically significant.

\section{Results}

\section{Literature selection and study characteristics}

According to the search strategy and inclusion and exclusion criteria, a total of six documents were included in this meta-analysis (see Fig. 1). Two studies researched ViV and ViR patients, one study included ViR and ViMAC patients, and three studies simultaneously researched ViV, ViR and ViMAC patients. In these studies, Mackram [25], Yoon [20], Jasleen [27] and Matheus [28] all achieved a complete follow-up of 30 days. Additionally, Yoon [20] and Matheus [28] also analysed the possible influencing factors of all-cause mortality. These factors were of great significance for evaluating the postoperative efficacy of TMVR in the absence of a randomized controlled trial in the field. Tables 1 and 2 shows detailed baseline characteristics as well as the number of deaths in each group and number of major adverse events. After reading the full text carefully, we used the NOS scoring standard to score the included literature. For the question of COMPARABILITY, we gave the literature two points when the researchers analysed other problems in addition to the main complications. Regarding the second question in OUTCOME, we believed that if the follow-up time was within 6 months, it was not long enough, and no points would be given. In the end, the highest score of our included literature was 9 points, the lowest was 7 points, and the overall quality was high. The results are shown in Fig. 2.

\section{Comparison of baseline characteristics}

Before the quantitative meta-analysis, we conducted a comparative analysis of the baseline characteristics of 


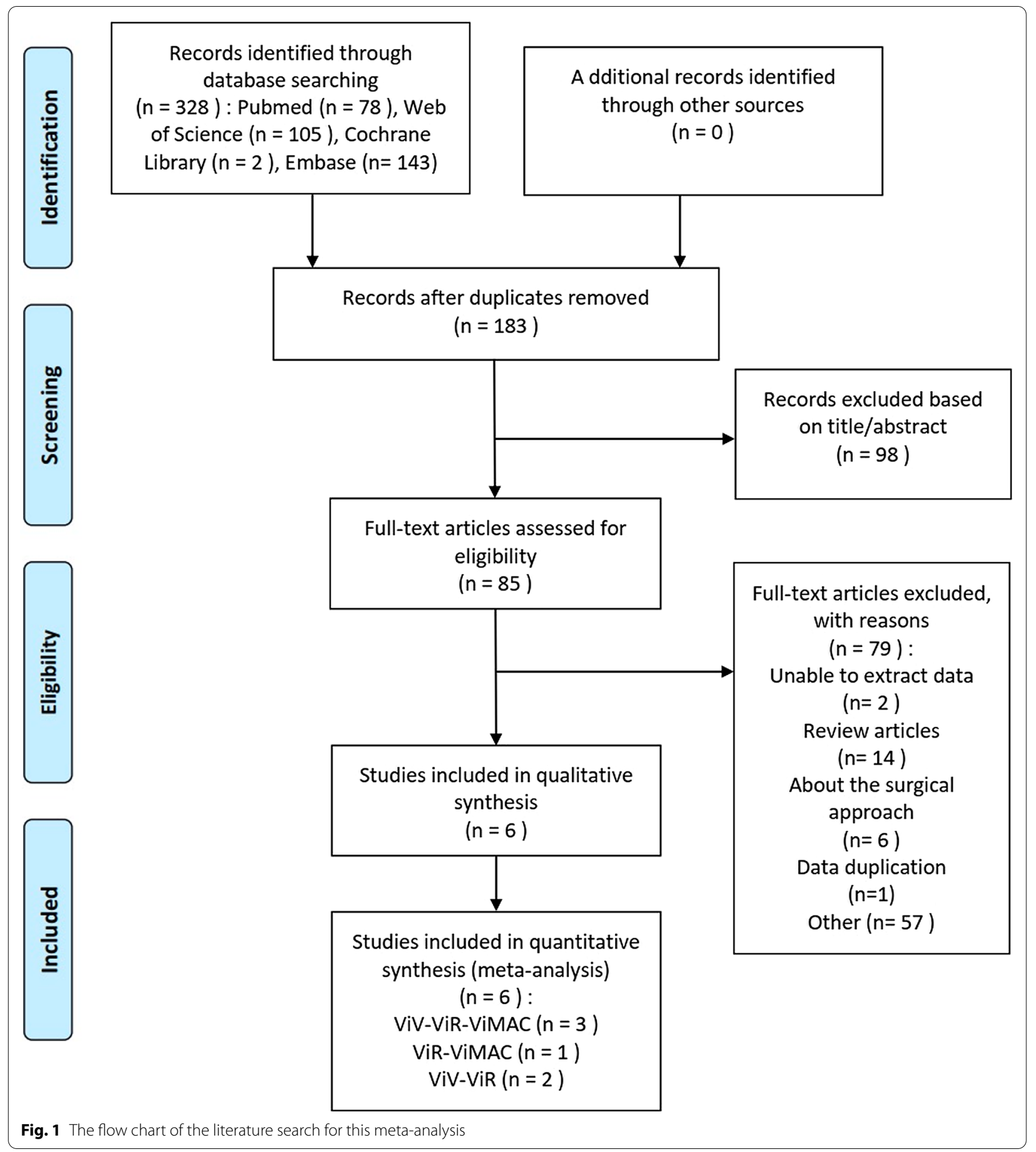

the included studies. $\mathrm{P}(\mathrm{Z})$ marked in bold italic indicates that there was a difference, and italic indicates that there was no difference.

The main differences in the inclusion of the population in the ViV-ViR group were age $(\mathrm{OR}=2.78,95 \%$ CI: $1.23-3.72)$, proportion of females $(\mathrm{OR}=1.73,95 \%$
CI: 1.44-2.10), number of patients with atrial fibrillation $(\mathrm{OR}=1.47,95 \% \mathrm{CI}: 1.18-1.84)$, creatinine value of patients (SMD $=-0.19,95 \% \mathrm{CI}:-0.38-0.01)$, and number of patients with previous cerebrovascular accidents $(\mathrm{OR}=1.59,95 \% \mathrm{CI}: 1.19-2.13)$. In addition, the creatinine value of the ViV group was lower than that of the 


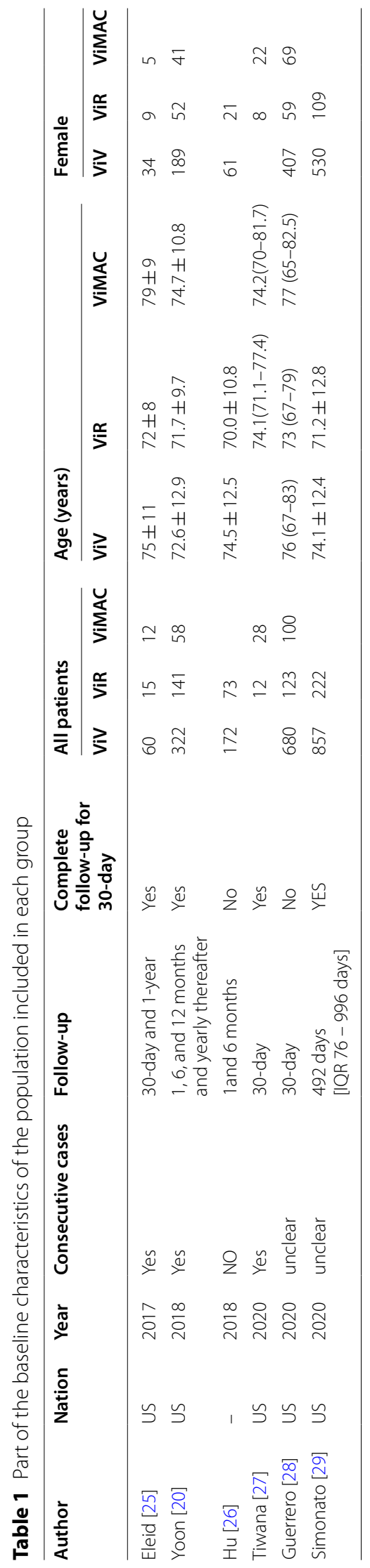




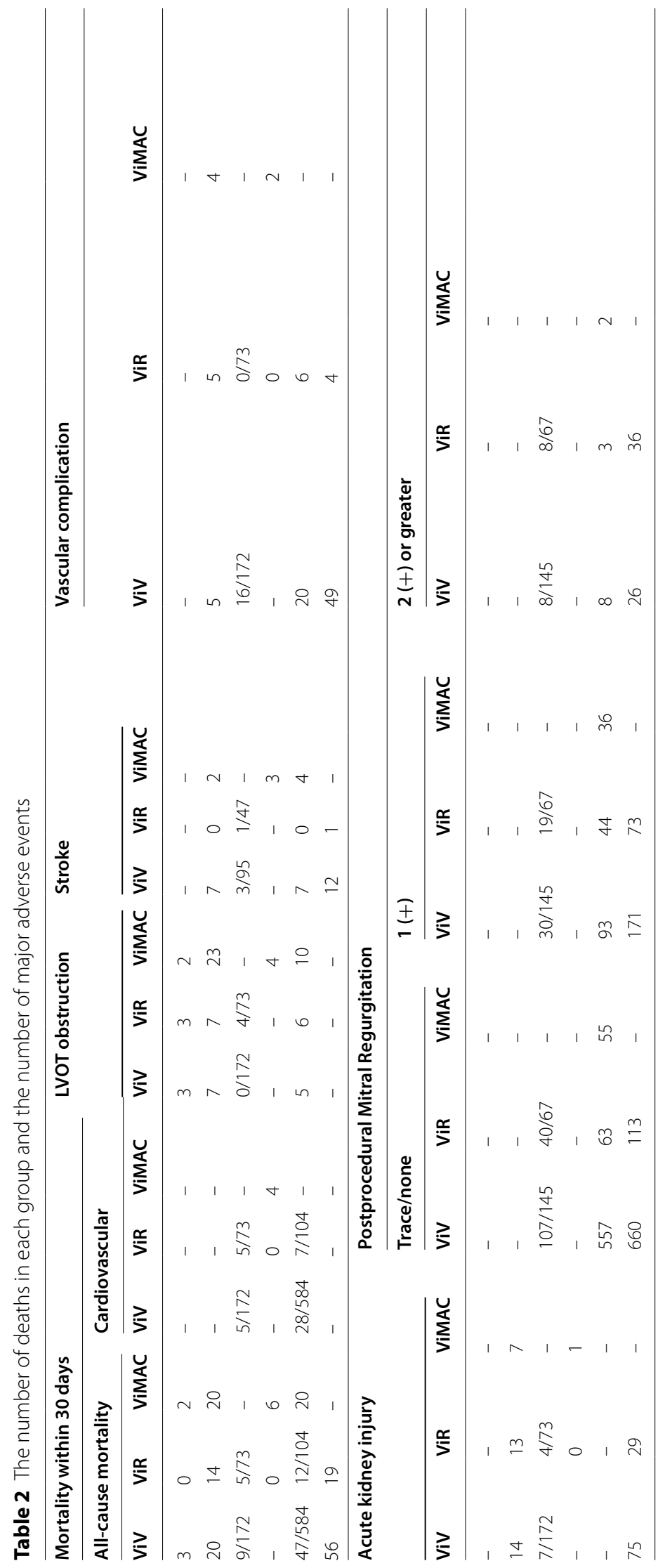




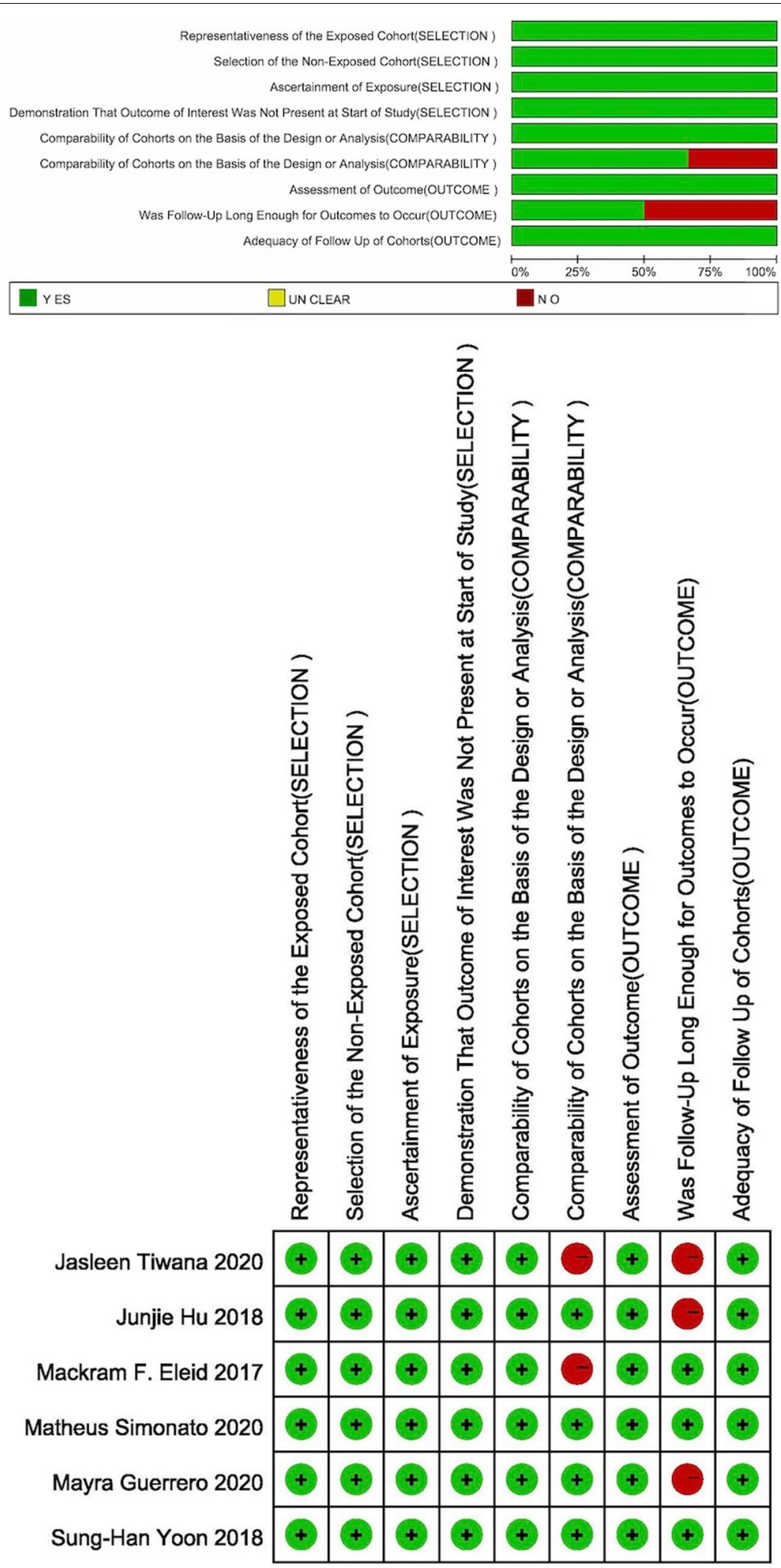

Fig. 2 The NOS score result of the included literature output by Revman 
ViR group (SMD $=-0.19,95 \% \mathrm{CI}:-0.38$ to -0.01$)$. For mode of progress failure, the patients in the ViV group showed more stenosis $(\mathrm{OR}=2.69,95 \% \mathrm{CI}$ : $1.50-4.80)$ and combined (OR $=1.56,95 \% \mathrm{CI}$ : $1.22-1.99)$, while the patients in ViR group had more regurgitation $(\mathrm{OR}=0.25$, 95\% CI: 0.16-0.40).

Similarly, we found some differences in the ViMACViR group: the patients in the ViMAC group were 3.78 years older than those in the ViR group (95\% CI: 0.91-6.66), and there were more women in the ViMAC group (OR $=2.21,95 \% \mathrm{CI}: 1.11-4.40)$ and patients with diabetes mellitus $(\mathrm{OR}=1.60,95 \% \mathrm{CI}$ : $1.07-2.38)$. In addition, many patients in the ViMAC group had undergone TAVR surgery, which was 9.74 times that in the ViR group (95\% CI: 2.54-37.36). However, the number of patients in the ViR group was greater than that in the ViMAC group, and the measured NT-proBNP value was also lower than that in the ViMAC group. These two values suggested that the heart condition of patients in the ViR group might be worse.

The results are shown in Tables 3 and 4 and Additional file 1: Figs. S1-S10.

\section{Meta-analysis of outcomes ViV versus ViR}

A total of five studies were included in this group. The ViV group included 2091 patients, and the ViR group included 574 patients. The following results were obtained by analysing the extracted data: the all-cause mortality within 30 days of the $\mathrm{ViV}$ group was lower than that of the ViR group, and the result was statistically significant $[\mathrm{OR}=0.70,95 \% \mathrm{CI}(0.50-0.99), P=0.04]$. The left ventricular ejection fraction (LVEF) after the operation in the ViV group was significantly higher than that in the ViR group $[\mathrm{MD}=8.74,95 \% \mathrm{CI}$ (7.03-10.46), $P<0.01]$, and the number of postprocedural trace/ no mitral regurgitations was significantly higher than that in the ViR group [OR=3.26, 95\% CI $(2.59-4.09)$, $P<0.01]$. Life-threatening or fatal events $[\mathrm{OR}=0.44,95 \%$ CI $(0.25-0.77), P<0.01]$ and embolization $[\mathrm{OR}=0.34$, $95 \%$ CI $(0.12-0.98), P=0.05]$ were more likely to occur in the ViR group after surgery. Comparing the two groups, the probability of conversion to cardiac surgery in the ViR group $[\mathrm{OR}=0.31,95 \% \mathrm{CI}(0.13-0.74), P=0.01]$ and secondary valve implantation $[\mathrm{OR}=0.21,95 \% \mathrm{CI}(0.13-$ $0.33), P<0.01]$ was also higher. At the same time, the VIR group was more prone to left ventricular outflow tract obstruction (LVOT) $[\mathrm{OR}=0.22,95 \%$ CI $(0.11-0.44)$, $P<0.01]$. The measured value of the mean Mitral valve gradient was slightly higher than that of the $\mathrm{ViV}$ group $[\mathrm{MD}=-0.46,95 \% \mathrm{CI}(-0.77-0.15), P<0.01]$. In addition, the incidence of acute kidney injury (AKI) in the VIR group increased [OR $=0.60,95 \%$ CI $(0.41-0.87)$,
$P<0.01]$, postprocedural $1(+)[\mathrm{OR}=0.45,95 \% \mathrm{CI}(0.35-$ $0.00), P<0.01]$ and $2(+)$ or greater $[\mathrm{OR}=0.22,95 \% \mathrm{CI}$ (0.14-0.35), $P<0.01]$. Mitral regurgitation occurred more frequently. The other outcomes were not statistically significant, $P>0.05$. The results are shown in Table 5 and Additional file 1: Figs. S10-S16.

\section{ViMAC versus ViR}

A total of four studies were included in this group, of which ViMAC included 198 patients and ViR included 291 patients. After analysing the data by RevMan 5.4, the results of all-cause mortality within 30 days $[\mathrm{OR}=2.95$, 95\% CI (1.76-4.93), $P<0.01$ ], stroke [OR $=5.16,95 \% \mathrm{CI}$ (1.14-23.38), $P=0.03]$ and LVEF $[\mathrm{MD}=13.64,95 \% \mathrm{CI}$ (10.02-17.26), $P<0.01]$ of the ViMAC group were all significantly higher than those of the ViR group, $P<0.05$. The other outcomes were not statistically significant, $P>0.05$. The results are shown in Table 6 and Additional file 1: Figs. S17-S21.

\section{Indirect comparison}

According to the different analysis results of the ViVViR and ViMAC-ViR groups, we further conducted an indirect comparative analysis between the TMViV and TMViMAC groups. Compared with the ViR group, the all-cause mortality within 30 days of the ViV group decreased by 0.3 . The probability of embolization, LVOT obstruction, conversion to cardiac surgery and need for second valve implantations decreased by $0.66,0.78,0.69$ and 0.79 , respectively. Mortality in the ViMAC group increased by 2.20 , and the other probabilities increased by $0.92,2.01,0.38$ and $0.06(P<0.05)$. Regarding postoperative trace/no mitral regurgitation, there were more in the ViV and ViMAC groups than the ViR group. According to the statistical analysis results, they were 2.16 and 0.17 higher than the ViR, respectively. No significant statistical results were found in outcome indicators such as blending, stroke, vascular composition or acute kidney injury. The results are shown in Fig. 3.

\section{Publication bias}

Since only six articles $(<10)$ were included in the final collection, we did not evaluate publication bias [30].

\section{Discussion}

Transcatheter mitral valve replacement (TMVR) seems to have become a viable option for patients with severe mitral valve disease, such as severe mitral valve bioprosthesis degradation, failure of valvuloplasty or severe mitral valve natural annulus calcification. Moreover, a certain clinical effect can be obtained, and the probability of complications has also been declining with the 


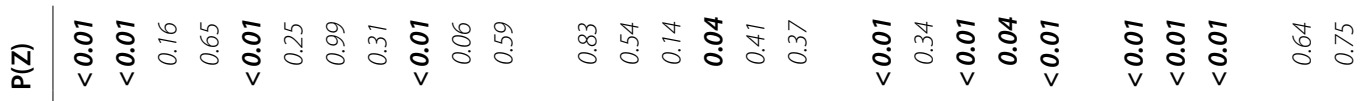

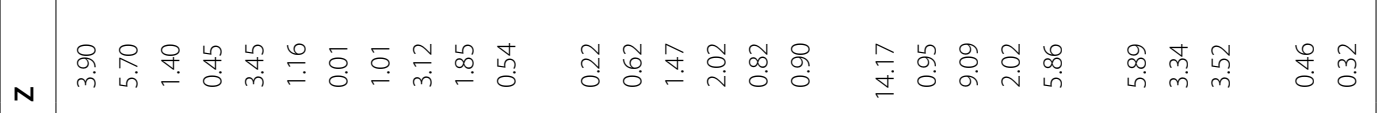
믐

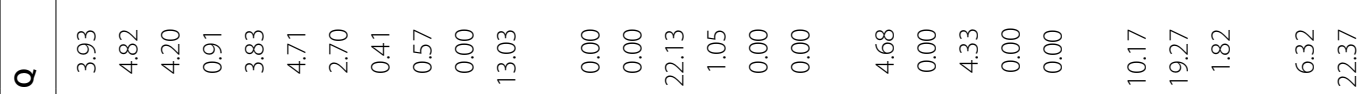

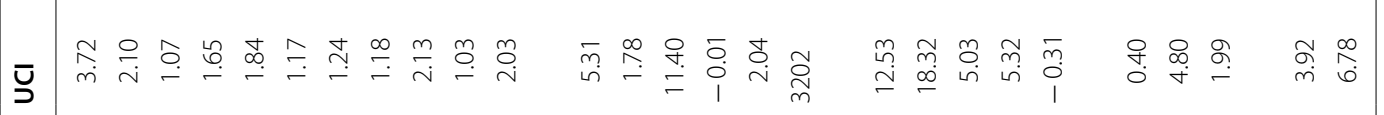

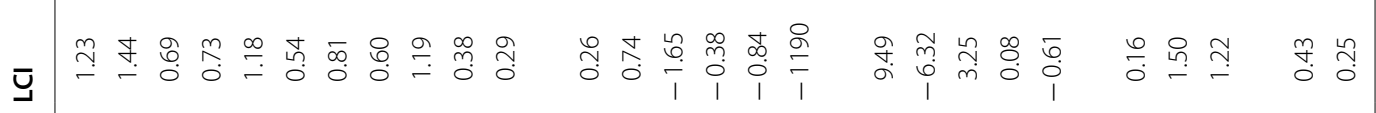

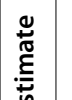

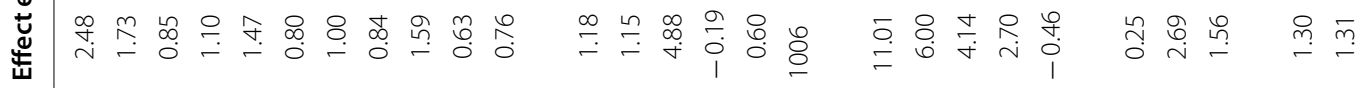

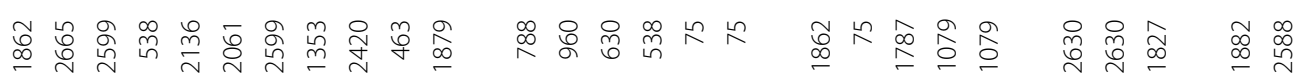

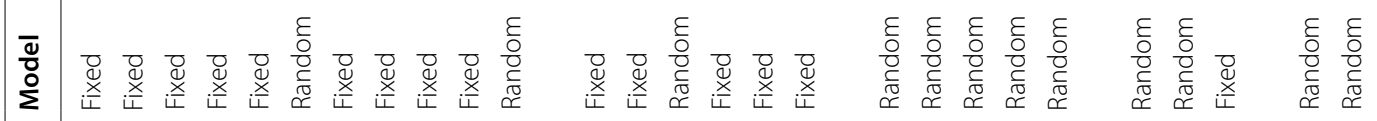

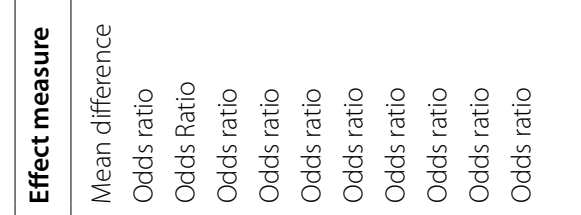

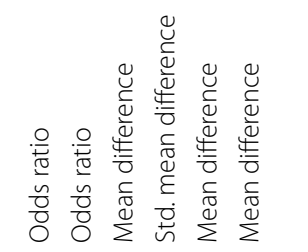

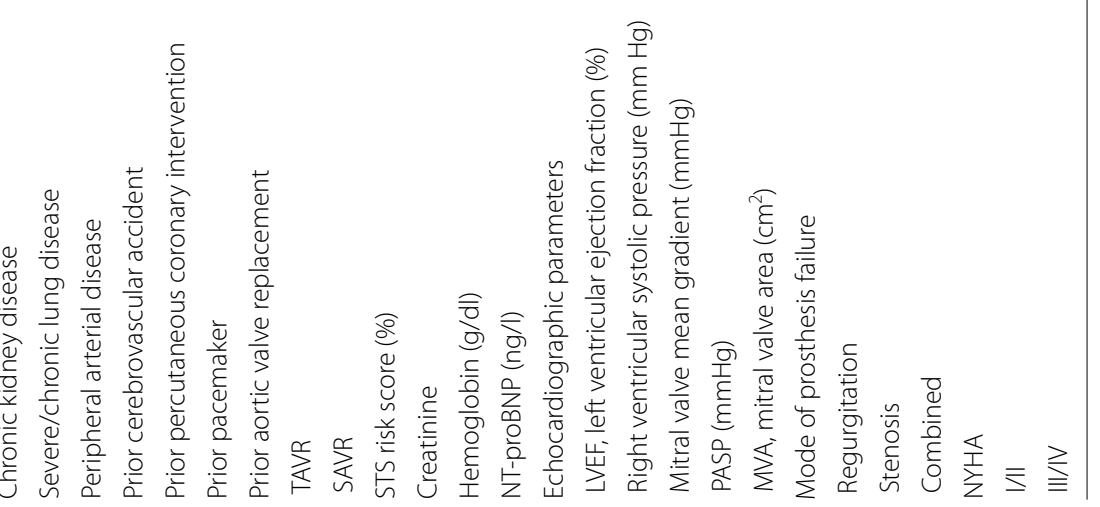




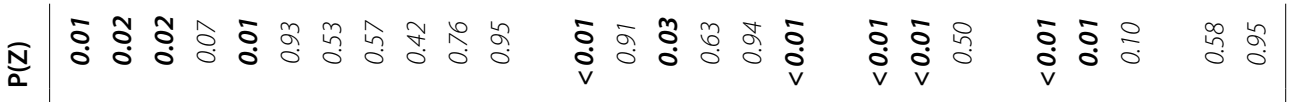

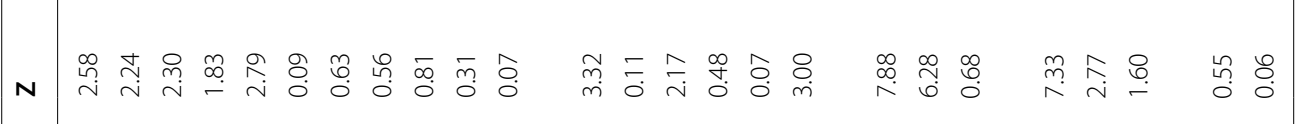

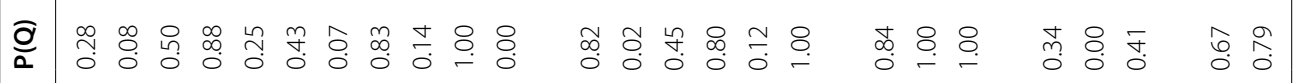

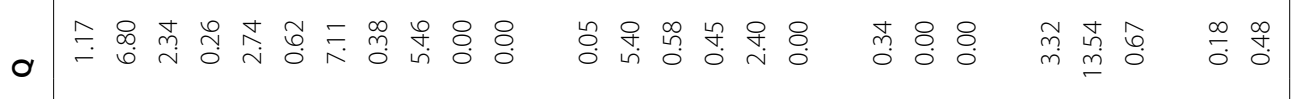

క ஏ ভ

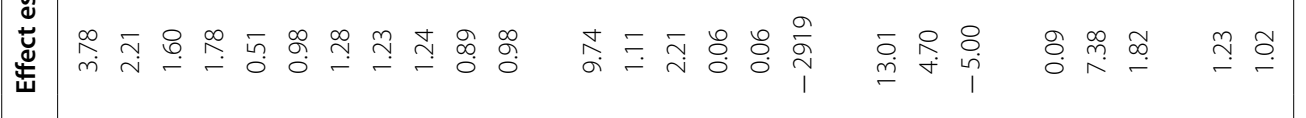

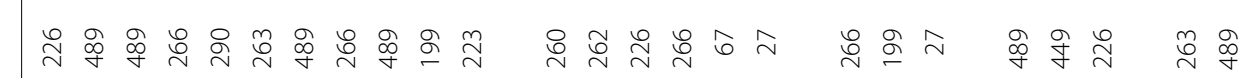

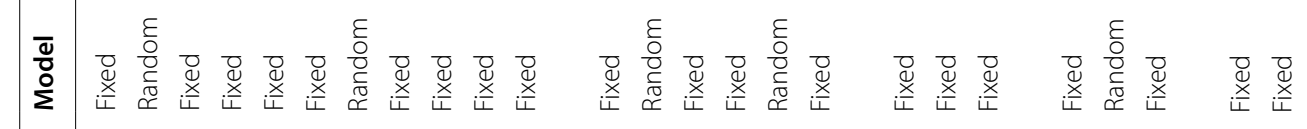

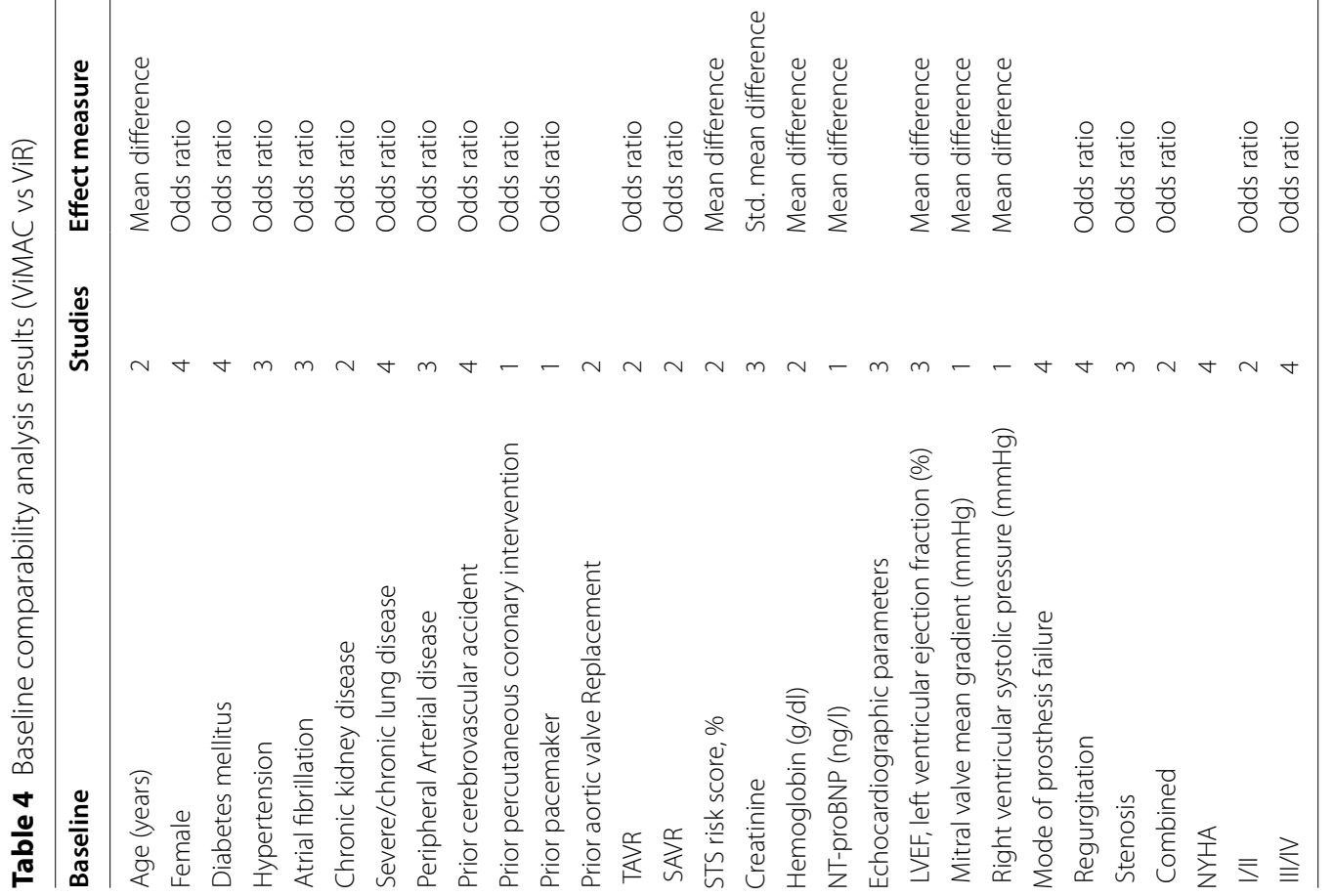




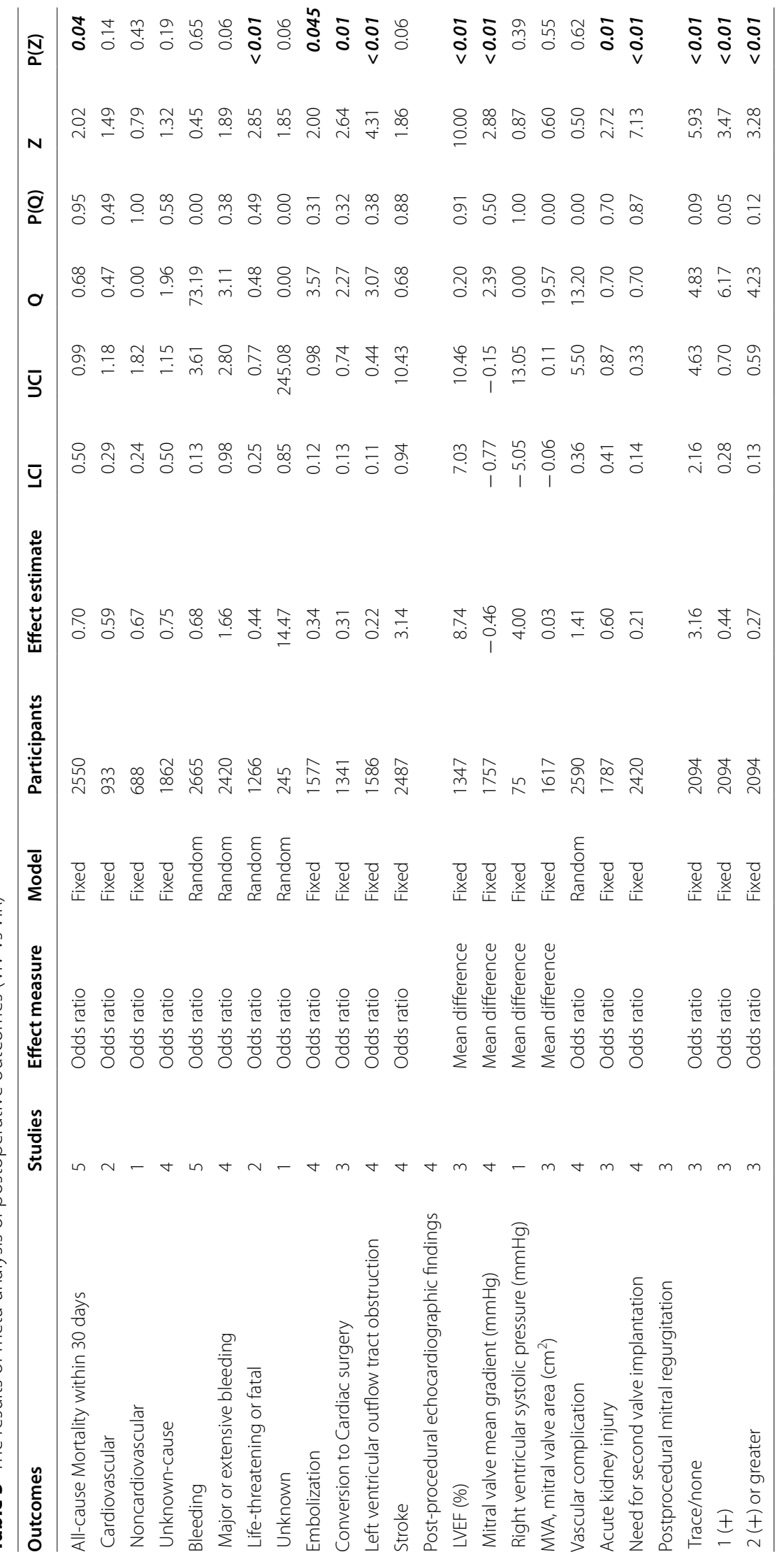




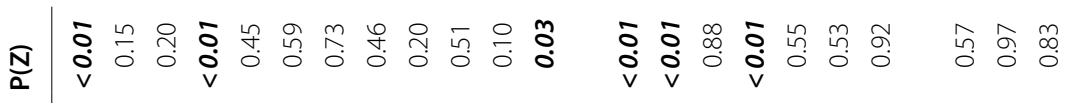

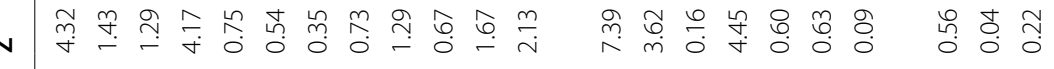

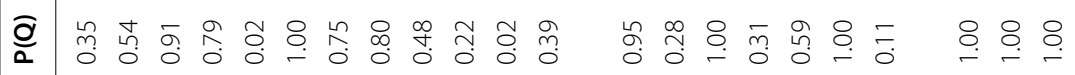

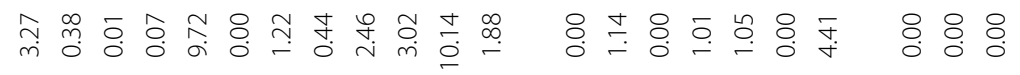

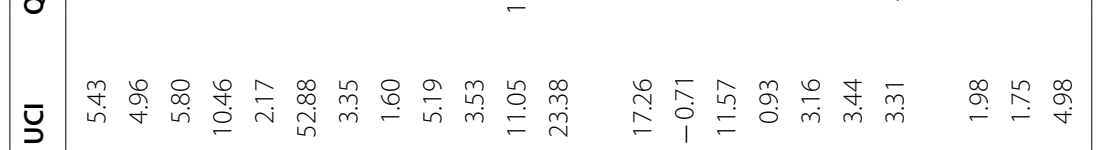
ఫ

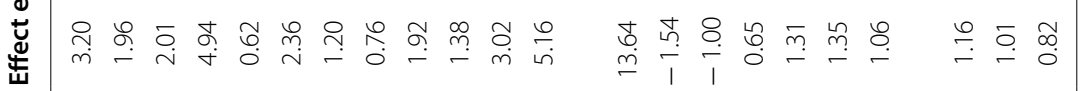

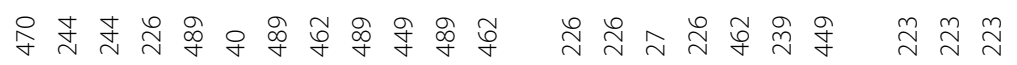

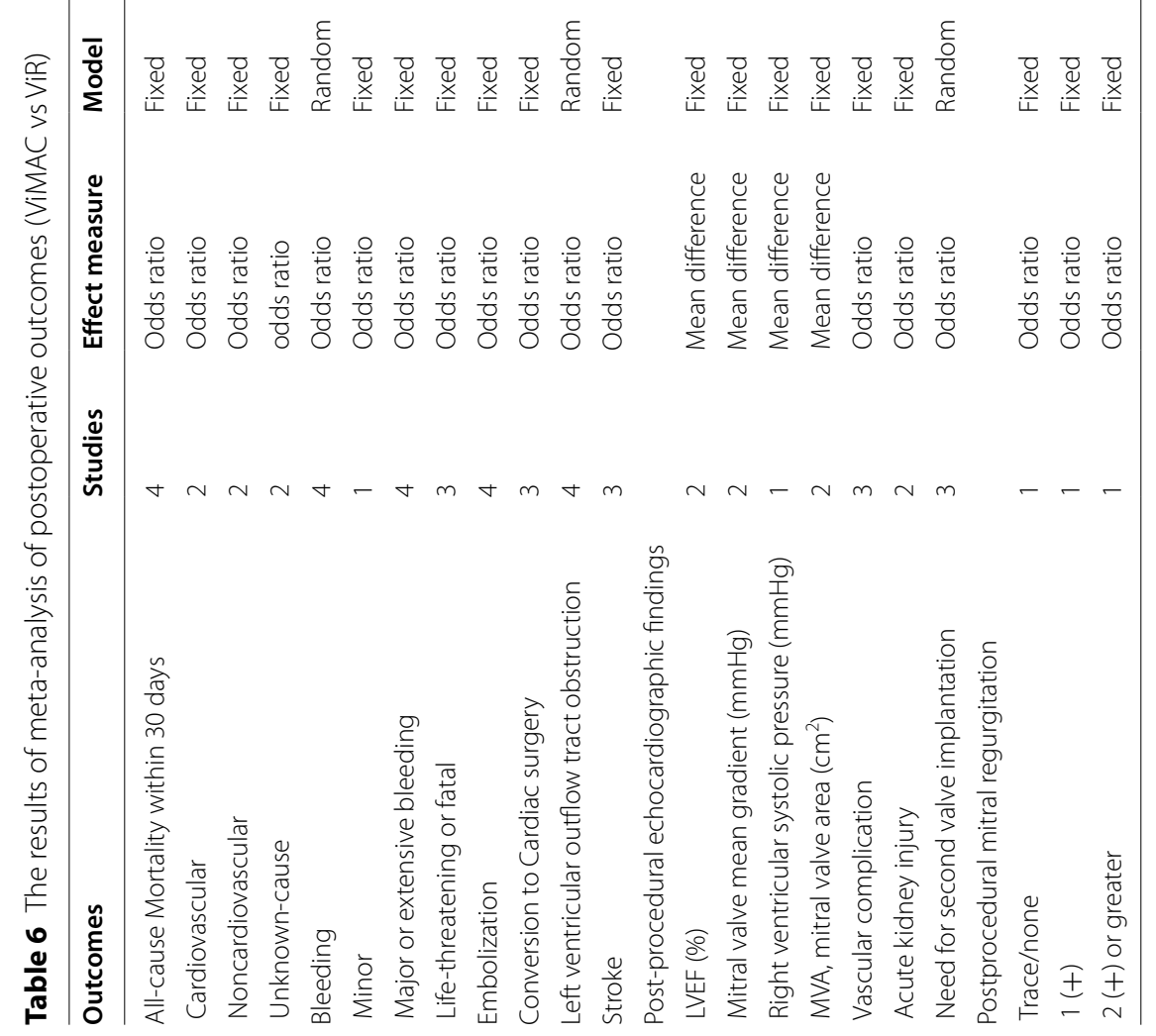




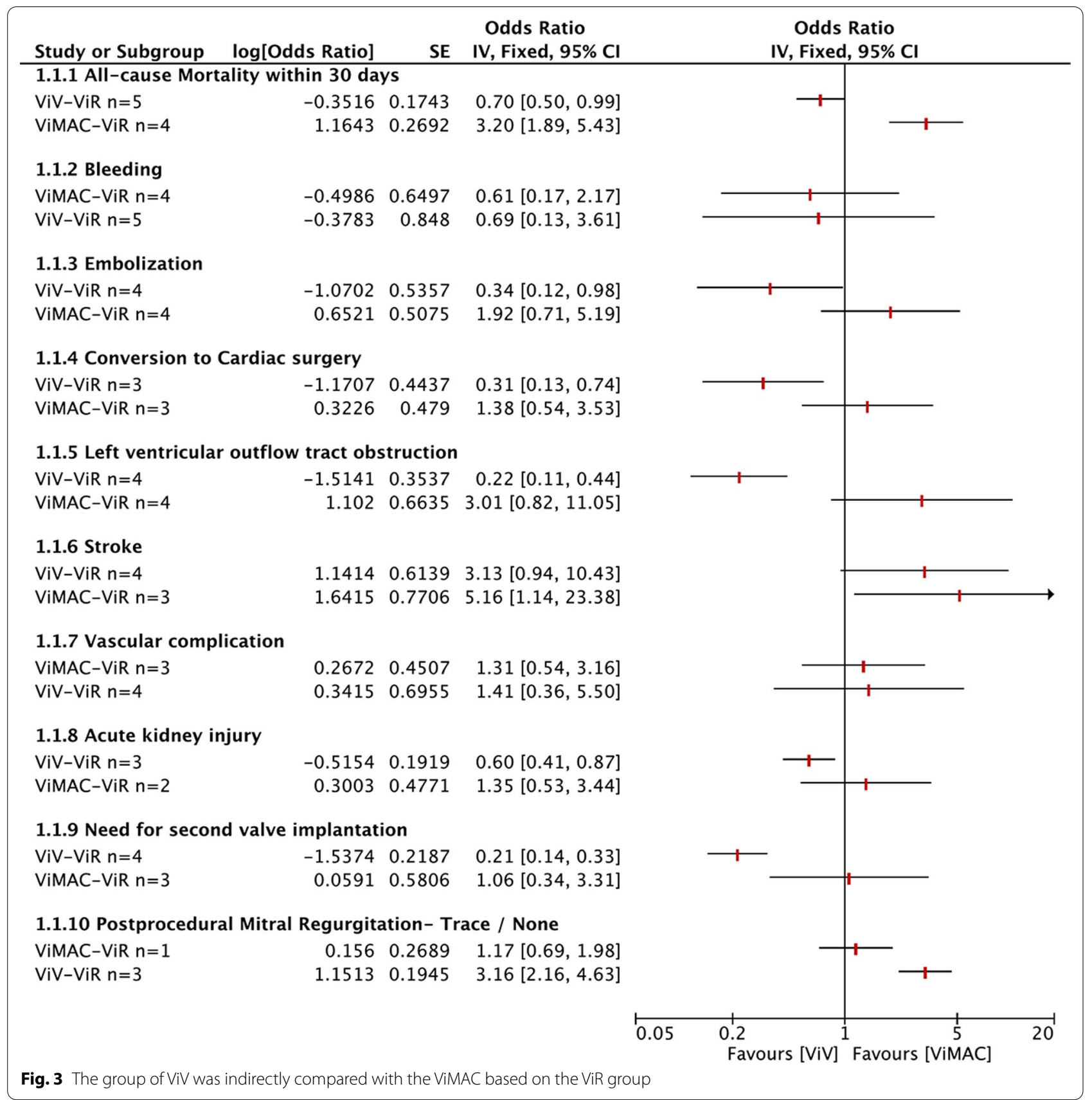

progress of surgical methods and experience of doctors [14]. We conducted this comprehensive and systematic review and meta-analysis to promote and disseminate these technologies in clinical diagnosis and treatment and enhance their value.

In this meta-analysis, we found that some of the baseline information in the $\mathrm{ViV}-\mathrm{ViR}$ group was incomparable, such as age, atrial fibrillation and previous cerebrovascular accidents. However, when analysing the impact of this baseline information on these two groups, the differences indicated that the situation of the ViV group was worse. The average age of patients in the ViV group was 2.48 years older than that in the ViR group, and the number of patients in the ViV group with atrial fibrillation was 1.47 times that of the ViR group. Nevertheless, TMViV still showed good applicability. The all-cause mortality within 30 days of the ViV group was lower than that of the ViR group, which was also proven in many large sample studies [20, 25, 28]. In addition, the analysis results indicated that the incidence of acute 
kidney injury in the ViR group was significantly greater than that in the ViV group. However, in terms of allcause mortality, Yoon [20] and Matheus Simonato's [29] prediction analysis of death factors showed that the two most important factors were "TMViR" and "Chronic Kidney Disease". In addition, the ViV group also showed better results in other clinical outcomes, such as a smaller probability of nonfatal haemorrhage and lower incidence of left ventricular outflow tract obstruction and embolism. Moreover, the probability of the ViV group being converted to cardiac surgery due to accidents during the treatment process was also less than that of the ViR group, which was more in line with our original intention of using a transcatheter for revalvular surgery. At the same time, the possibility of high-grade mitral valve postoperative residual regurgitation in the $\mathrm{ViV}$ group was far less than that in the ViR group. In contrast, it was more likely to be trace/none. This also proved the feasibility of transcatheter surgery in patients with mitral valve bioprostheses (TMViVs).

Compared with TMViR, TMViMAC had higher shortterm mortality when applied to patients with severe MAC. The same conclusion was reached in some multicentre joint studies. Among the patients with high surgical risk (advanced age or higher risk score of the Society of Thoracic Surgeons, etc.), the 30-day all-cause mortality of TMViMAC was very high. Patients at special risk, such as patients with chronic lung disease, chronic renal insufficiency and high ejection fraction, might require multiple valve operations [31-34]. From the comparison of baseline information, age might be a potential explanation for the higher mortality rate in the ViMAC group. However, the higher number of atrial fibrillations (ViMAC vs. ViR: $\mathrm{OR}=0.51$ ) in the ViR group and the higher probability of heart failure (NT-proBNP, ViMAC vs. ViR: $M D<0$ ) indicated that the situation of the ViR group was worse. Although the results of LVOT obstruction in the ViR-ViMAC group obtained by this metaanalysis were not statistically significant $(P=0.10)$, the incidence of patients in the ViMAC group was significantly higher than that in the ViR group. Coupled with the lower incidence of stroke in the ViR group after surgery, we had more reason to believe that TMViR was better than TMViMAC.

Based on the ViR group, through indirect analysis, the ViV group had the lowest all-cause mortality within 30 days, which might benefit from fewer complications and a lower incidence of residual mitral regurgitation. The lower the incidence of LVOT obstruction was, the smaller the possibility of switching to traditional heart surgery and replacing the valve again. All this evidence allowed us to see its excellent effects. It was suggested that TMViV might become the first-line treatment method for the treatment of mitral valve bioprostheses. Regarding the high incidence of embolization in the three groups, long-term postoperative anticoagulation therapy might be a better approach [35]. For some relatively poor outcomes of ViMAC, existing studies believe that this might be related to more basic information and comorbidities, such as age, sex, diabetes and renal impairment [36-38]. However, a more decisive factor might be the baseline CT-MAC calcium score [36]. The higher the patient's MAC baseline was, the higher the disease activity and the faster the progression. It also reflected the vicious calcium cycle that was established in the patient's body, which further calcified the mitral valve. Even so, TMVR is still a viable alternative treatment option for some inoperable severe MAC patients [27, 31, 32]. Perhaps surgical mitral valve replacement utilizing a transcatheter aortic valve in the mitral position (MVRTAVR) used by Joseph would be another viable option for patients with severe MAC [39].

At present, the better applicability of TMViV, TMViR and TMViMAC has been proven by many studies, including some multicentre clinical research results $[25,28,29,32]$. It was undeniable that these operations still had some common serious complications, such as LVOT obstruction, bleeding and acute kidney injury. Although there were various difficulties, scholars in various countries were actively looking for solutions, for instance, improving postoperative outcomes through surgical approaches. In a study by Eleid [40], transfemoral percutaneous venous mitral valve implantation in patients with a high risk of bioprosthesis degradation was safe and effective. It was also conducive to rapid improvement of haemodynamics and functional status. For patients with annuloplasty ring failure and severe MAC, further studies are needed in view of the high short-term morbidity and mortality feasibility. Transseptal TMVR, which eliminates the need for extracorporeal circulation and naturally reduces the risk, is considered a safer route to perform repeat valve surgery $[19,41]$. It has the advantages of less invasiveness, does not require opening the chest and avoids trauma to the left ventricle. Because of this, it is more popular with patients and clinicians [42]. In addition, in terms of the Achilles heel of TMVR, there have been increasing studies on iatrogenic LVOT obstruction, which was defined as an LVOT peak gradient increase of $\geq 10 \mathrm{mmHg}$ post-TMVR. [43-46]. The incidence of LVOT obstruction in TMVR occurs in up to $10-40 \%$ of ViMAC, $5 \%$ of ViR, and $0.7-2 \%$ of $\mathrm{ViV}$ cases [20, $28]$. Once it happens, the result will be very poor, and the hospital mortality rate may be as high as $62 \%$ [19]. 
Therefore, it was particularly important to study how to solve or avoid this problem. It is worth emphasizing that preventing the risk of LVOT obstruction was the key to improving the results. Among the published methods, the intentional transcatheter laceration of the anterior mitral valve leaflet (LAMPOON) technique and alcohol septal ablation (ASA) are considered effective [45, 47]. At the same time, it has also been well proven in the research of Khan [48] that LAMPOON could effectively prevent LVOT obstruction from TMVR. However, neither of these two technologies has been approved by the Food and Drug Administration (FDA). Perhaps in the future, large sample data could provide different results.

Although a more detailed comparison and analysis were performed, there were still certain limitations. First, there were few studies available for analysis, especially the study on TMViMAC, and the accuracy of the results would be affected. Second, due to the follow-up time and content of different studies, we only obtained an early result. However, the overall quality of the included literature was relatively high, and the results were relatively reliable. Third, some baselines were incomparable, but the baseline information all had a positive impact, which was opposite to the direction of the outcome indicators. Such results suggest that better patient baseline information will increase the credibility of the outcome. In addition, there was a lack of evidence for a direct comparison between TMViV and TMViMAC in the included studies, but we conducted an indirect comparison this time to answer the pros and cons of these two procedures. Considering these limitations, the results of this meta-analysis need to be interpreted carefully, and we look forward to better randomizing clinical trial comparison models in the future to further prove the feasibility of TMViV, TMViR and TMViMAC.

\section{Conclusion}

In summary, the existing evidence shows that TMViV and TMViMAC have lower mortality and complication rates, which are favoured by many patients. TMVR showed promise for patients with severe MAC, and further studies are needed to prove its feasibility.

\footnotetext{
Abbreviations

ViV: Valve-in-valve; ViR: Valve-in-ring; ViMAC: Valve-in-MAC; MAC: Mitral annulus calcification; AHA: American Heart Association; TMVR: Transcatheter mitral valve replacement; TMViV: Transcatheter mitral valve-in-valve; TMViR: Transcatheter mitral valve-in-ring; TMViMAC: Transcatheter mitral valve-inMAC; NOS: Newcastle-Ottawa Scale; OR: Odds ratio; MD: Mean difference; SMD: Std. mean difference; LVEF: Left ventricular ejection fraction; LVOT: Left ventricular outflow tract; TAVR: Transcatheter aortic valve replacement; SAVR: Surgical aortic valve replacement; MVR-TAVR: Mitral valve replacement
}

utilizing a transcatheter aortic valve in the mitral position; FDA: Food and Drug Administration.

\section{Supplementary Information}

The online version contains supplementary material available at https://doi. org/10.1186/s13019-021-01677-7.

Additional file 1: Fig. S1. Comparison of baseline information between the group of ViV and ViR. The type of data analyzed was Dichotomous using the Random effects. Fig. S2. Comparison of baseline information between the group of ViV and ViR. The type of data analyzed was Dichotomous using the Fixed effects. Fig. S3. Comparison of baseline information between the group of ViV and ViR. The type of data analyzed was Continuous (MD) using the Random effects. Fig. S4. Comparison of baseline information between the group of ViV and ViR. The type of data analyzed was Continuous (MD) using the Fixed effects. Fig. S5. Comparison of baseline information between the group of ViV and ViR. The type of data analyzed was Continuous (SMD) using the Fixed effects. Fig. S6. Comparison of baseline information between the group of ViMAC and ViR. The type of data analyzed was Dichotomous using the Random effects. Fig. S7. Comparison of baseline information between the group of ViMAC and ViR. The type of data analyzed was Dichotomous using the Fixed effects. Fig. S8. Comparison of baseline information between the group of ViMAC and ViR. The type of data analyzed was Continuous (MD) using the Random effects. Fig. S9. Comparison of baseline information between the group of ViMAC and ViR. The type of data analyzed was Continuous (MD) using the Fixed effects. Fig. S10. Comparison of baseline information between the group of ViMAC and ViR. The type of data analyzed was Continuous (SMD) using the Fixed effects. Fig. S11. Comparison of Outcomes between the group of ViV and ViR. The type of data analyzed was Dichotomous using the Random effects. Fig. S12. Subgroup of Bleeding between the group of ViV and ViR. The type of data analyzed was Dichotomous using the Random effects. Fig. S13. Comparison of Outcomes between the group of ViV and ViR. The type of data analyzed was Dichotomous using the Fixed effects. Fig. S14. Subgroup of all-cause mortality within 30 days between the group of ViV and ViR. The type of data analyzed was Dichotomous using the Fixed effects. Fig. S15. Comparison of Outcomes between the group of ViV and ViR. The type of data analyzed was Continuous (MD) using the Fixed effects. Fig. S16. Comparison of Outcomes between the group of ViV and ViR. The type of data analyzed was Continuous (SMD) using the Fixed effects. Fig. S17. Comparison of Outcomes between the group of ViMAC and ViR. The type of data analyzed was Dichotomous using the Random effects. Fig. S18. Subgroup of Bleeding between the group of ViMAC and ViR. The type of data analyzed was Dichotomous using the Fixed effects. Fig. S19. Comparison of Outcomes between the group of ViMAC and ViR. The type of data analyzed was Dichotomous using the Fixed effects. Fig. S20. Subgroup of all-cause mortality within 30 days between the group of ViMAC and ViR. The type of data analyzed was Dichotomous using the Fixed effects. Fig. S21. Comparison of Outcomes between the group of ViMAC and ViR. The type of data analyzed was Continuous (MD) using the Fixed effects.

\section{Acknowledgements}

The authors gratefully acknowledge the financial supports by the Natural Science Foundation of Gansu Province (Project Number: 21JR1RA027) and Health industry scientific research project of Gansu Province (GSWSKY2016-04).

\section{Authors' contributions}

Conceptualization: KY, WW, TY. Data collection: WW, YM, XZ, JG. Formal analysis: TY, WW, SH, XX, XL. Funding acquisition: TY. Investigation: TY, WW, JG, SH, XL. Methodology: KY, WW, YM, TY. Software: TY, WW. Validation: KY, WW, TY. Supervision: WW, TY. Writing—original draft:TY, WW. Writing—review and editing: TY, WW, KY, XZ, JG, SH, XX, YM, XL. All authors read and approved the final manuscript.

\section{Funding}

Natural Science Foundation of Gansu Province (21JR1RA027); Health industry scientific research project of Gansu Province (GSWSKY2016-04). 


\section{Availability of data and materials}

All data generated or analysed during this study are included in this published article and its supplementary information files.

\section{Declarations}

Ethics approval and consent to participate

All analyses will be based on previously published studies; thus, no ethical approval and consent to participate are required.

\section{Consent for publication}

Not applicable.

\section{Competing interests}

The authors declared that there have no competing interests.

\section{Author details}

'Department of Cardiovascular Surgery, Gansu Provincial Hospital, No. 204, Donggang West Road, Lanzhou City 730000, Gansu Province, China. ${ }^{2}$ Gansu International Scientific and Technological Cooperation Base of Diagnosis and Treatment of Congenital Heart Disease, Lanzhou, China. ${ }^{3}$ The First Clinical Medical College of Lanzhou University, Lanzhou, China. ${ }^{4}$ The First School of Clinical Medical of Gansu University of Chinese Medicine, Lanzhou, China.

${ }^{5}$ The Second Clinical Medical College of Lanzhou University, Lanzhou, China.

Received: 21 July 2021 Accepted: 28 September 2021

Published online: 10 October 2021

\section{References}

1. Virani SS, Alonso A, Aparicio HJ, et al. Heart Disease and Stroke Statistics-2021 update: a report from the American Heart Association. Circulation. 2021;143(8):e254-743.

2. Goldstone AB, Chiu P, Baiocchi M, et al. Mechanical or biologic prostheses for aortic-valve and mitral-valve replacement. N Engl J Med. 2017;377(19):1847-57.

3. Mohty D, OrszulakTA, Schaff HV, Avierinos JF, Tajik JA, Enriquez-Sarano M. Very long-term survival and durability of mitral valve repair for mitral valve prolapse. Circulation. 2001;104:11-7.

4. Beute TJ, Goehler M, Parker J, Boeve T, Heiser J, Murphy E, TimekT, Willekes CL. Long-term outcomes of mosaic versus perimount mitral replacements: 17-year follow-up of 940 implants. Ann Thorac Surg. 2020;110:508-15.

5. David TE, Armstrong S, McCrindle BW, Manlhiot C. Late outcomes of mitral valve repair for mitral regurgitation due to degenerative disease. Circulation. 2013;127:1485-92.

6. Lazam S, Vanoverschelde JL, Tribouilloy C, Grigioni F, Suri RM, Avierinos JF, De MC, Barbieri A, Rusinaru D, Russo A, et al. Twenty-year outcome after mitral repair versus replacement for severe degenerative mitral regurgitation: analysis of a large, prospective, multicenter. International Registry Circulation. 2017;135:410-22.

7. Albeyoglu SC, Filizcan U, Sargin M, Cakmak M, Goksel O, Bayserke O, Cinar B, Eren E. Determinants of hospital mortality after repeat mitral valve surgery for rheumatic mitral valve disease. Thorac Cardiovasc Surg. 2006;54:244-9.

8. Jamieson WR, Burr LH, Miyagishima RT, Janusz MT, Fradet GJ, Lichtenstein SV, Ling H. Reoperation for bioprosthetic mitral structural failure: risk assessment. Circulation. 2003;108(Suppl 1):I198-102.

9. Vohra HA, Whistance RN, Roubelakis A, Burton A, Barlow CW, Tsang GM, Livesey SA, Ohri SK. Outcome after redo-mitral valve replacement in adult patients: a 10-year single-centre experience. Interact Cardiovasc Thorac Surg. 2012;14:575-9.

10. Toker ME, Eren E, Guler M, Kirali K, Yanartas M, Balkanay M, Yakut C. Second and third cardiac valve reoperations: factors influencing death and long-term survival. Tex Heart Inst J. 2009;36:557-62.

11. Expósito V, García-Camarero T, Bernal JM, Arnáiz E, Sarralde A, García I, Berrazueta JR, Revuelta JM. Repeat mitral valve replacement: 30-years' experience. Rev Esp Cardiol. 2009;62:929-32.
12. Webb JG, Cheung AW, Dvir D. Transcatheter mitral valve replacement when mitral surgery fails: 10 years later. J Am Coll Cardiol. 2017:70(9):1132-4.

13. Dvir D, Webb JG. Mitral valve-in-valve and valve-in-ring: technical aspects and procedural outcomes. Eurolntervention. 2016;12:93-6.

14. Grover FL, Vemulapalli S, Carroll JD, et al. 2016 annual report of the society of thoracic surgeons/American College of Cardiology transcatheter valve therapy registry. J Am Coll Cardiol. 2017;69(10):1215-30.

15. Paradis JM, Del Trigo M, Puri R, Rodes-CJ. Transcatheter valve-in-valve and valve-in-ring for treating aortic and mitral surgical prosthetic dysfunction. J Am Coll Cardiol. 2015;66:2019-37.

16. Yoon S-H, Whisenant BK, Bleiziffer $\mathrm{S}$, et al. Transcatheter mitral valve replacement for degenerated bioprosthetic valves and failed annuloplasty rings. J Am Coll Cardiol. 2017;70:1121-31.

17. Cheung A, Webb JG, Wong DR, et al. Transapical transcatheter mitral valve-in-valve implantation in a human. Ann Thorac Surg. 2009;87:18-20.

18. de Weger A, Ewe SH, Delgado V, et al. First-in-man implantation of a trans-catheter aortic valve in a mitral annuloplasty ring: novel treatment modality for failed mitral valve repair. Eur J Cardiothorac Surg. 2011:39:1054-6.

19. Guerrero M, Urena M, Himbert D, et al. 1-Year outcomes of transcatheter mitral valve replacement in patients with severe mitral annular calcification. J Am Coll Cardiol. 2018;71(17):1841-53.

20. Yoon SH, Whisenant BK, Bleiziffer S, et al. Outcomes of transcatheter mitral valve replacement for degenerated bioprostheses, failed annuloplasty rings, and mitral annular calcification. Eur Heart J. 2019:40(5):441-51.

21. Vohra HA, Whistance RN, Bezuska L, Livesey SA. Surgery for non-rheumatic calcific mitral stenosis. J Heart Valve Dis. 2011:20:624-6.

22. Casarotto D, Bortolotti U, Thiene G, Gallucci V, Cévese PG. Rupture of the posterior wall of the left ventricule after replacement of the mitral valve: a description of 8 cases (author's transl). G Ital Cardiol. 1977;7:387-94.

23. Stang A. Critical evaluation of the Newcastle-Ottawa scale for the assessment of the quality of nonrandomized studies in meta-analyses. Eur J Epidemiol. 2010;25(9):603-5.

24. Wells G. The Newcastle-Ottawa Scale (NOS) for assessing the quality of non-randomised studies in meta-analyses. In: Symposium on systematic reviews: beyond the basics. 2014.

25. Eleid MF, Whisenant BK, Cabalka AK, et al. Early outcomes of percutaneous transvenous transseptal transcatheter valve implantation in failed bioprosthetic mitral valves, ring annuloplasty, and severe mitral annular calcification. JACC Cardiovasc Interv. 2017;10(19):1932-42.

26. Hu J, Chen Y, Cheng S, et al. Transcatheter mitral valve implantation for degenerated mitral bioprostheses or failed surgical annuloplasty rings: a systematic review and meta-analysis. J Card Surg. 2018;33(9):508-19.

27. Tiwana J, Aldea G, Levin DB, et al. Contemporary transcatheter mitral valve replacement for mitral annular calcification or ring. JACC CardiovasC Interv. 2020;13(20):2388-98.

28. Guerrero M, Vemulapalli S, Xiang $Q$, et al. Thirty-day outcomes of transcatheter mitral valve replacement for degenerated mitral bioprostheses (valve-in-valve), failed surgical rings (valve-in-ring), and native valve with severe mitral annular calcification (valve-in-mitral annular calcification) in the united states: data from the society of thoracic Surgeons/American College of Cardiology/Transcatheter Valve Therapy Registry. Circ Cardiovasc Interv. 2020;13(3):e008425.

29. Simonato $M$, Whisenant $B$, Ribeiro HB, Webb JG, Kornowski R, Guerrero M, Wijeysundera $\mathrm{H}$, Søndergaard L, De Backer O, et al. Transcatheter mitral valve replacement after surgical repair or replacement: comprehensive midterm evaluation of valve-in-valve and valve-in-ring implantation from the VIVID registry. Circulation. 2021;143:104-16.

30. Sterne JA, Egger M, Smith GD. Systematic reviews in health care: Investigating and dealing with publication and other biases in meta-analysis. BMJ. 2001;323(7304):101-5.

31. Praz F, Khalique OK, Lee $R$, et al. Transatrial implantation of a transcatheter heart valve for severe mitral annular calcification. J Thorac Cardiovasc Surg. 2018;156(1):132-42.

32. Guerrero M, Dvir D, Himbert D, et al. Transcatheter mitral valve replacement in native mitral valve disease with severe mitral annular calcification: results from the first multicenter global registry. JACC CardiovasC Interv. 2016;9(13):1361-71. 
33. Kanjanauthai S, Nasir K, Katz R, et al. Relationships of mitral annular calcification to cardiovascular risk factors: the Multi-Ethnic Study of Atherosclerosis (MESA). Atherosclerosis. 2010;213(2):558-62.

34. Chan V, Ruel M, Hynes M, Chaudry S, Mesana TG. Impact of mitral annular calcification on early and late outcomes following mitral valve repair of myxomatous degeneration. Interact Cardiovasc Thorac Surg. 2013;17(1):120-5.

35. Maisano F, Taramasso M. Mitral valve-in-valve, valve-in-ring, and valve-inMAC: the good, the bad, and the ugly. Eur Heart J. 2019;40(5):452-5.

36. Massera D, Trivieri MG, Andrews JPM, et al. Disease activity in mitral annular calcification. Circ Cardiovasc Imaging. 2019;12(2):e008513.

37. Boon A, Cheriex E, Lodder J, et al. Cardiac valve calcification: characteristics of patients with calcification of the mitral annulus or aortic valve. Heart. 1997:78:472-4.

38. Nair CK, Sudhakaran C, Aronow WS, et al. Clinical characteristics of patients younger than 60 years with mitral anular calcium: comparison with age- and sex-matched control subjects. Am J Cardiol. 1984;54:1286-7.

39. Lamelas J, Alnajar A. Early outcomes for surgical minimally invasive SAPIEN 3 transcatheter mitral valve replacement. Ann Thorac Surg. 2020;112:494-500.

40. Eleid MF, Cabalka AK, Williams MR, et al. Percutaneous transvenous transseptal transcatheter valve implantation in failed bioprosthetic mitral valves, ring annuloplasty, and severe mitral annular calcification. JACC Cardiovasc Interv. 2016;9(11):1161-74

41. Nazir S, Lohani S, Tachamo N, et al. Outcomes following transcatheter transseptal versus transapical mitral valve-in-valve and valve-in-ring procedures. J Cardiovasc Thorac Res. 2018;10(4):182-6.

42. Dvir D. Transseptal instead of transapical valve implantation: making mitral great again? JACC Cardiovasc Interv. 2016;9:1175-7.
43. Wang $D D$, Eng $M H$, Greenbaum $A B$, et al. Validating a prediction modeling tool for left ventricular outflow tract (LVOT) obstruction after transcatheter mitral valve replacement (TMVR). Catheter Cardiovasc Interv. 2018;92:379-87.

44. Khan JM, Babaliaros VC, Greenbaum AB, et al. Anterior leaflet laceration to prevent ventricular outflow tract obstruction during transcatheter mitral valve replacement. J Am Coll Cardiol. 2019;73:2521-34.

45. Harloff MT, Chowdhury M, Hirji SA, et al. A step-by-step guide to transseptal valve-in-valve transcatheter mitral valve replacement. Ann Cardiothorac Surg. 2021;10(1):113-21.

46. Wang DD, Guerrero M, Eng MH, et al. Alcohol septal ablation to prevent left ventricular outflow tract obstruction during transcatheter mitral valve replacement: first-in-man study. JACC Cardiovasc Interv. 2019:12(13):1268-79.

47. Babaliaros VC, Greenbaum AB, Khan JM, et al. Intentional percutaneous laceration of the anterior mitral leaflet to prevent outflow obstruction during transcatheter mitral valve replacement: first-in-human experience. JACC Cardiovasc Interv. 2017;10:798-809.

48. Khan JM, Babaliaros VC, Greenbaum AB, et al. Anterior leaflet laceration to prevent ventricular outflow tract obstruction during transcatheter mitral valve replacement [published correction appears in J Am Coll Cardiol. 2019 Jul 30;74(4):595]. J Am Coll Cardiol. 2019;73(20):2521-34.

\section{Publisher's Note}

Springer Nature remains neutral with regard to jurisdictional claims in published maps and institutional affiliations.
Ready to submit your research? Choose BMC and benefit from:

- fast, convenient online submission

- thorough peer review by experienced researchers in your field

- rapid publication on acceptance

- support for research data, including large and complex data types

- gold Open Access which fosters wider collaboration and increased citations

- maximum visibility for your research: over $100 \mathrm{M}$ website views per year

At $\mathrm{BMC}$, research is always in progress.

Learn more biomedcentral.com/submissions 